\title{
Astronomy Classification: Towards a Faceted Classification
} Scheme

\begin{tabular}{|r|l|}
\hline Journal: & Knowledge Organization \\
\hline Manuscript ID & KO-2019-0023.R2 \\
\hline Manuscript Type: & Article \\
\hline
\end{tabular}

\section{SCHOLARONE $^{\text {M }}$ Manuscripts}


Astronomy Classification: Towards a Faceted Classification Scheme

\begin{abstract}
Astronomy classification is often overlooked in classification discourse. Its rarity and obscurity, especially within UK librarianship, suggests it is an underdeveloped strand of classification research and is possibly, undervalued in modern librarianship. The purpose of this research is to investigate the suitability and practicalities of the discipline of astronomy adopting a subject-specific faceted classification scheme and to provide a provisional outline of a special faceted astronomy classification scheme. The research demonstrates that the application of universal schemes for astronomy classification had left the interdisciplinary subject ill catered for and outdated; making accurate classification difficult for specialist astronomy collections. A faceted approach to
\end{abstract}


classification development is supported by two qualitative literature-based research methods; historical research into astronomy classification and an analytico-synthetic classification case study. The subsequent classification development is influenced through a pragmatic and scholarly-scientific approach and constructed by means of instruction from faceted classification guides by Vickery (1960) and Batley (2005), and faceted classification principles from Ranaganathan (1937). This research fills a gap within classification discourse on specialist interdisciplinary subjects, specifically within astronomy and demonstrates the best means for their classification. It provides a means of assessing further the value of faceted classification within astronomy librarianship.

1. Introduction

\subsection{Background}

Corbin states that $(2003,145)$ : "astronomers have a dependence on the librarian to make needed information accessible". Librarians are the key to providing the means of access to these versatile collections. As well as providing catalogues in which to search for astronomical information, classification of these materials provides the secondary access point which enables information retrieval and location. It is with this access in mind that this research provides its practicality by creating a means of classifying astronomy collections through a new subject-specific classification scheme. 
The scientific discourse of researchers in the field of astronomy is of some interest to knowledge organization researchers, for example Ibekwe-SanJuan's (2008) research revealed the role that geographic location can play in the development of the field and also in the distribution of terminology. In relation to bibliographic classification, Corbin $(2003,142)$ found that most special astronomy libraries classify their materials either using an in-house scheme, developed specifically for their collections, or sub-sections of universal classification schemes. Globally, the three most common classification schemes used in astronomy libraries are Library of Congress Classification (LCC), Dewey Decimal Classification (DDC) and Universal Decimal Classification (UDC) (Corbin 2003, 142). While there are no comprehensive astronomy classification schemes to date, there have been previous attempts to create a scheme. The Physics and Astronomy Classification Scheme (PACS), developed by the American Institute of Physics (AIP), was used to classify online journals and indexes, databases, and astronomy and physics catalogues (Hider and Harvey 2008, 126), before it was replaced by the AIP Thesaurus (Access Innovation Inc. 2018). The move from the PACS to the AIP Thesaurus has left an opening within astronomy classification for a new comprehensive astronomy scheme.

A review into discourse on classification theory emphasises two main classification types; enumerative, which have a one dimensional top-down organisational approach with predefined notation (Losee 1995), and faceted, which are broken down into their constituent parts starting with the subject fields (Broughton 2015) and further divided by 'facets' with unset notation (Chowdhury and Chowdhury 2007). There is ambiguity surrounding the term 'analytico-synthetic classification'; the term originating as a synonym for 'faceted classification'. La Barre (2006), discussing the historical development of faceted analytico-synthetic theory (FAST), notes that while work on FAST 
began in the 1930s, it was not codified until Ranganathan published Prolegomena to Library Science in 1957. Her findings, which showed how FAST underpins the design of informational and promotional websites, demonstrates the ongoing usefulness and significance of FAST in the digital information age. Recent literature by Chowdhury and Chowdhury (2007), Chowdhury (2010) and Broughton (2015) identifies analyticosynthetic as a new classification type, containing both enumerative and faceted features allowing the content of an item to be split into its component parts (analysed; enumerative) and then a class mark to be built from the notation of each part (synthesised; faceted). Therefore, this study defines analytico-synthetic as separate from enumerative and faceted schemes.

A review into why special subject-specific classifications are built produced the following quote by Vickery $(1960,7)$, summing up the enduring reasoning behind the process: "Several reasons may be given why existing general schemes are unsatisfactory. First, most of them do not give adequate detail for accurate specification of the highly complex subjects in papers and reports that documentation must handle today. Second, despite the comprehensiveness and variety of certain general schemes, they do not fully cater for the special viewpoints of each particular library or information centre. Third, even if they are varied in viewpoint, they do not sufficiently provide for the flexible combination of terms which highly specific subject headings demand. Fourth, even if flexible, they achieve such flexibility only by unnecessarily lengthy or complicated notational means. Fifth, they fail to give optimum helpfulness in filing order." This perspective is supported by Vickery (1960) and Herner and Meyer (1957) when discussing how quickly evolving and complex subject areas have outgrown universal classification schemes. 
Herner and Meyer $(1957,801)$ found seven requirements for the creation of a specialist classification scheme. 1 . terms used must reflect the current use of language within the subject area (hospitable). 2. the scheme must be suitable for the type and purpose of literature (flexible). 3. terms and classes used must be distinguishable in meaning and content (unique). 4. classification structure should allow for equal distribution of documents over an easy to see structure (simple). 5. the scheme must cater for the addition of new subject matter (hospitable). 6. notation must be consistent and easily recognised and deduced (brevity, mnemonic). 7. the scheme must group comparable subjects together and use hierarchy for user needs (expressive). The requirements satisfy classification discourse (Berwick Sayers 1955; Chowdhury and Chowdhury 2007; Broughton 2015; Hunter 2018) in that notations need to assume the following qualities; expressiveness, mnemonics, simplicity, uniqueness, brevity, flexibility and hospitality.

\subsection{Purpose of Research}

The purpose of this research is to investigate the suitability and practicalities of the discipline of astronomy adopting a subject-specific faceted classification scheme and offers the beginnings of such a specialist scheme. Faceted science classifications have become popular due to their flexibility and ease of development. Traditional schemes provide a means of classifying universal knowledge, making them time consuming to update and hard to specify. The creation of a specific classification scheme using faceted principles requires minimal time to build (in comparison to a general revision) and will be adaptable to new astronomy topics. This research contributes to the literature in developing the foundations of an astronomy classification using faceted classification principles. It is hoped that this initial study might lead to further development. 


\section{Methodology}

\subsection{Research Methods}

A qualitative methodology is employed in the form of historical research and a case study, influencing and forming the foundation of the faceted classification development. The historical research into the development and use of astronomy notation within universal classification schemes is undertaken using thematic analysis of conference proceedings and practical evaluation of primary classification schedules. The conference proceedings were from the Astronomical Society of the Pacific Conference Series; the Library and Information Services in Astronomy (LISA) conferences, ranging from LISA I (1988) to VIII (2017) and the Astronomical Data Analysis Software and Systems (ADASS) conferences, ranging from ADASS XV (2005) to XXIII (2013). All articles were chosen based on their topic applicability within astronomy classification with a total of ten analysed thematically. Classification schemes offering astronomy notation are analysed within the context of their suitability in providing detailed subject-specific notation and this highlights the issues of astronomy notation creation. The literature-based case study of INSPEC, a faceted/analytico-synthetic physics classification, involving the review and analysis of INSPEC schedules and secondary literature is undertaken to examine whether interdisciplinary subjects are suited to faceted library classifications. The schemes notational qualities are analysed using an analytical framework based on Berwick Sayers' $(1955,60)$ ideal qualities of classification schemes. The case study highlights the suitability of faceted classification principles being adopted in interdisciplinary subjects and provides a grounded classification example for the faceted astronomy classification. The application of the research findings contributes to the development of the faceted astronomy classification. 


\subsection{Analysis of Findings and Classification Development}

The findings of the historical research are analysed thematically from the grouping of codes, comprising of the types of astronomy classification; LCC, DDC, UDC, Dewhirst, and astronomy classification tools; IAU Thesaurus, AIP Thesaurus and the Unified Astronomy Thesaurus (UAT). The findings of the case study are analysed thematically from Berwick Sayers ideals of classification. Both research methods are analysed separately, providing layers of reasoning, supporting the purpose of this study in helping to form the basis of classification development. The final outcome of the study is the design of a provisional faceted astronomy classification. This development is broken down into its constituent parts; faceted classification principles, classification design including discipline, vocabulary, notation, examples and provisional classification. The development highlights the applicability of faceted principles to the discipline of astronomy and provides a means of further development after the conclusion of this study.

\subsection{Ethical Considerations}

This research is governed by the ethical code of conduct laid out by the British Sociological Association (2002) statement of ethical practice and Aberystwyth University's ethical guidelines. Any information of a sensitive nature is dealt with the necessary amount of data protection required for the information, as influenced by the BSA and the CILIP (2015) code of professional practice.

\section{Historical Research}

\subsection{Background}

Astronomy, an educational and research subject, has evolved slowly since its transformation in the middle ages, from a tool for creating calendars to understanding 
our place in the solar system with Copernicus' heliocentric model (Hoskin 2003). Diversification of astronomy came in the late $20^{\text {th }}$ century, where new technology and observational techniques expanded the subject at an ever-increasing rate. Crovisier and Intner (1987) observed that instead of being the tightly compacted and well-organised science of the $19^{\text {th }}$ century, astronomy grew into a loosely defined and broad subject area. It is this change which has enabled astronomy to outgrow the larger universal classification schemes and to warrant its own subject-specific scheme.

\subsection{Astronomy coverage in universal classification}

The LCC table for astronomy is represented by the beginning notation QB within Class Q (science). Its structure is based on the original 1905 scheme with little change implemented through the six revisions up until the 1980's (Crovisier and Intner 1987). The schedules development was based on the publication of new monographs which was reliant on research published in journal papers. It is this delay in revision which hinders the schedules ability to be truly up-to-date and to fully integrate new subject developments. The enumerative qualities of this scheme were highly unsuited for the interdisciplinary subject in the late 1980's, with the various areas of astronomy dispersed amongst the other Class Q tables such as physics and geology (Crovisier and Intner 1987). This concept scattering occurs in most enumerative schemes and is seen in the example topic of 'planetary geology'. In older versions of the LCC, works discussing the geology of the inner terrestrial planets would sometimes be placed in QE (geology) instead of QB (astronomy) due to their emphasis on physical terrestrial landforms (Crovisier and Intner 1987). As of modern-day, the QB table has been revised to include certain interdisciplinary subjects such as QB455-456 astrogeology. This is not a catch-all solution 
where interdisciplinary topics are classed in $Q B$; its implementation is reliant on the librarian's subject understanding and nature of collections.

The DDC schedule for astronomy is represented by the beginning notation 520 within the 500 Class (science). The structure of the 520 table has changed very little in the last 20 years as can be seen by comparing the $21^{\text {st }}$ edition to the WebDewey version. The main change is the addition of objects to existing subfields i.e. 523.4 Planets $=523.4$ Planets, asteroids, trans-Neptunian objects of the solar system. The issues that arose within the LCC schedules are apparent in DDC and is mainly to do with the set structure of the enumerative classification type. Rowley and Hartley $(2008,209)$ found that the application of faceted principles to DDC has made the scheme much more flexible in notation building. This has allowed for better interdisciplinary classification but has limited use within the astronomy schedule due to its subject specificity.

The creation of UDC from DDC schedules, and subsequent faceted revisions, has provided a fully universal analytico-synthetic scheme with the UDC table for astronomy represented by the beginning notation of 52 . The scheme underwent its first revision of the astronomy schedule in 1975 and a secondary revision in the 1990's (Wilkins 1989; 1995). It was found that sub-classes within the 52 class were outdated with some dropped for newer subjects. For example, in the 1975 revision, the 522/525/526 classes were cancelled and reissued for new use, with major changes taking place for classes 520/521/523 and 524 (Wilkins 1989). Even though changes were made, the scheme quickly became outdated again and the second revision focused on updating the whole discipline. Again, the focus was on classes 520 to 524, and this time Wilkins (1995) reported there was a call for astronomers, as experts, to help with the revision. The lack 
of expertise in schedule revision meant less was done to make the scheme truly suitable for astronomy classification, even though it was used within astronomy libraries (Wilkins 1989).

\subsection{Specialist astronomy classification tools}

The most well-known special astronomy classification, the Dewhirst classification (DC), was developed in the Institute of Astronomy at Cambridge University (Heck, 2003). The scheme is based on the classification used within Astronomy \& Astrophysics Abstracts, however, it was adapted to the library collections. The DC varies from the universal schemes as the schedules for astronomy were revised by a professional astronomer and librarian, thereby achieving the revision process attempted in UDC. The scheme itself has been revised four times, the most recent attempt in 2014. Its enumerative structure means that it is hard to apply new interdisciplinary topics to the already full denominations. A lack of literature makes it hard to assess its everyday practicality, however its main fault is a lack of brevity.

The IAU Thesaurus was developed in 1984 as a means of creating standardised astronomical terminology for cataloguing and was last revised between 1993 and 1995 (Lesteven et al. 2007). A further revision was undertaken in 2000 and resulted in the thesaurus' evolution into the International Virtual Observatory Alliance Thesaurus (Frey et al. 2015). This version of the thesaurus is still currently used and, as of 2017, has 2890 concepts (BARTOC.org 2017). The abandonment of the IAU thesaurus led to the development of the UAT, which is a current astronomy thesaurus that has real practical implications for astronomy classification. 
The UAT was developed to provide a free and community supported astronomy and astrophysics vocabulary which could be used by the astronomy community in the classification of journal articles and books (Accomazzi et al. 2014). The development of this thesaurus resulted from various outdated thesauri and vocabulary, such as the IAU and PACS, that were present in astronomical and astrophysical journals (Frey et al. 2015). The collaboration of physicists and astronomers to produce a unified thesaurus guaranteed the investment and development required for the thesaurus to be updated and used. The thesaurus can be searched alphabetically or hierarchically, with each entry showing narrower, broader and related terms.

The AIP Thesaurus is the remainder of the PACS. This classification tool was originally developed as a physics and astronomy classification scheme before it became unwieldy and was reduced to a more manageable thesaurus (Access Innovations Inc. 2018). It is currently used to help classify journal articles but it is no longer maintained. This classification tool demonstrates the life cycle of independent astronomy classification schemes and their inevitable disappearance due to a lack of expert guided revisions and funding.

\subsection{Astronomy Notation}

The following classification from LCC, DDC, UDC, DC and UAT schemes showcase their current ability to provide notation for the complex subject area of supernovae/supernovae remnants. Green and Jones $(2015,355)$ describe a supernova as "an outburst in which a star suddenly increases in brightness by an enormous factor $\left(\sim 10^{6}\right)$. Such a star is ending its life in a gigantic explosion from the collapse of its core". There are two main supernovae types, Type I and Type II, with Type I divided into three 
subtypes; Ia, Ib and Ic (Green and Jones 2015, 229). Any classification scheme must be able to provide for complete specification of supernovae types (i.e. Type Ib) or for material on the general subject matter of which supernovae is just one aspect (i.e. star evolution).

\section{LCC: QB 843.S95}

QB Astronomy

(The Library of Congress 2017)

495-903 Descriptive Astronomy

799-903 Stars

843. Other particular types of stars, A-Z

S95 Supernovae

Example 1: LCC

DDC: $\mathbf{5 2 3 . 8 4 4 6 5}$

(WebDewey 2017)

520 Astronomy

523 Specific Celestial Bodies \& Phenomena

523.8 Stars

523.84 Aggregations and variable stars

523.844 Variable stars

523.844 6 Eruptive variables

523.844 65 Supernovas

Example 2: DDC

UDC: $\mathbf{5 2 4 . 3 5 2}$

(British Standards Institution 2005)

52 Astronomy. Astrophysics. Space Research. Geodesy

524 Stars. Stellar Systems. The Universe

524.3 Stars

524.35 Supernovae and related objects. Peculiar stars

524.352 Supernovae

Example 3: UDC

DC: 122

(Institute of Astronomy 2017)

Group XI. Stars and the Galaxy

122. Supernovae. Supernovae Remnants

Example 4: DC

The LCC notation places the object type under descriptive astronomy, stars and other types of stars whilst DDC puts it under specific celestial bodies and phenomena, stars and variable objects. The UDC notation puts the object type under stars and then its own heading of supernovae and related objects whilst DC puts it under stars and the galaxy.

DC is the only scheme to mention both supernovae and supernovae remnants, even if 
they are detailed under the same notation. These schemes have trouble dividing the different elements of the object within a document meaning that all supernovae are lumped together under one notational category. In the case of LCC and DC, the broader category is stars, whilst UDC and DDC are the only schemes to provide a narrower category for supernovae. There is no further subdivision of the subject area into types and all the schemes are unable to cater for the differences between the process of producing supernovae and their aftermath i.e. supernovae remnants. The specificity that is lacking in these schemes can be achieved through the application of further notation, feasibly in the form of key terms or facets. In comparison, the UAT has supernovae as a narrower term for either stellar remnant or stellar type, providing a choice of both categories.

\section{UAT:}

(Unified Astronomy Thesaurus no date)

Stellar Astronomy

\section{Supernovae}

Broader Terms: Stellar Remnants - Stellar Types

Narrower Terms: Core-collapse supernovae - Hypernovae - Type Ia supernovae Related Terms: burst astrophysics - ejecta - supernova dynamics - white dwarf stars Example 5: UAT

The term supernovae is divided into the objects main types allowing for specification. The related terms help users searching within this subject area to find other related areas of research. Not all supernovae types are listed under the narrower terms, however, development of the thesaurus is still ongoing and this omission may be resolved in the future. The UAT displays the type of specificity which an astronomy classification would need to provide broad classificatory assistance.

\subsection{Findings}


The discourse on astronomy classification was limited as the main literature source came from the LISA conference proceedings with evidence found supporting the revision of the UDC and the UAT with the proviso to improve library classification for astronomy collections. Crovisier and Intner $(1987,32)$, whose work on the revision of LCC is central to the historical review, argue that "In classification, as in mechanics, inertia is a powerful agent against change". This statement defines the evolution of astronomy classification within universal classification schemes and their lack of consistent maintenance. These schemes have attempted to introduce specificity within their topic headings with the implementation of subject revisions and auxiliary tables, allowing for notation to be built with additional criteria, however their main purpose is for classifying universal knowledge thereby neglecting specificity. The only special astronomy classification that could be found currently employed was DC, yet even this scheme is lacking specificity as it is built around the collections rather than the discipline. It is with the development of a fully faceted scheme that the discipline of astronomy can find the specificity and flexibility needed to allow continuous revision without compromise to the whole scheme and complete control over notation building. Furthermore, the use of UAT, which has proven itself to be a flexible and reliable astronomical thesaurus, would provide sound descriptive elements and up-to-date terminology.

\section{Case Study: INSPEC}

\subsection{Geophysics, Astronomy and Astrophysics}

The creation of the INSPEC classification in 1969 revolutionized the way STEM subjects were viewed in universal classification schemes. These schemes realised the need to develop scientific areas to compete with the dominant humanities schedules. Originally INSPEC was created to provide journal classification enabling the searching of journal 
articles with its sectional classification schedules. The concordance to the INSPEC classification between 1969-1976 (INSPEC 1976) provides a lack of evidence of notational coding for the discipline of astronomy before 1973, with the first 'schedule' dedicated to astronomy and astrophysics found within the 1973 INSPEC interdisciplinary subjects schedule (Field 1973, 51).

\section{Z INTERDISCIPLINARY SUBJECTS}

ZM Astronomy and Astrophysics

ZMAAAF Astronomy and astrophysics

ZMBAAN Celestial mechanics

ZMCAAW Theoretical astrophysics

ZMEAAL Solar system

ZMGAAB Stars

ZMKAAZ Radio sources, infrared, $x$-ray and gammas-ray sources

ZMMAAP Galaxies, stellar systems

ZMRAAV Interstellar matter

ZMTAAK Astronomical measurements listed by type of observation ZMVAAS Astronomical techniques and instrumentation

Example 6: INSPEC Interdisciplinary subject schedule (Field 1973)

Within the initial schedules for the unified INSPEC classification, there was a limit of five hierarchical levels, to prevent excessive complexity. Each hierarchical level could contain more than ten subdivisions; so, to mitigate against loss of order each subdivision was allocated a six-letter code (Field 1973, viii). The sequence relied upon the number of A's to ascertain the hierarchical level, with no A's in the sequence indicating the narrowest hierarchy and four A's representing the broadest hierarchy, as seen in Example 7. The last letter in the sequence was always a check number (Field 1973, viii).

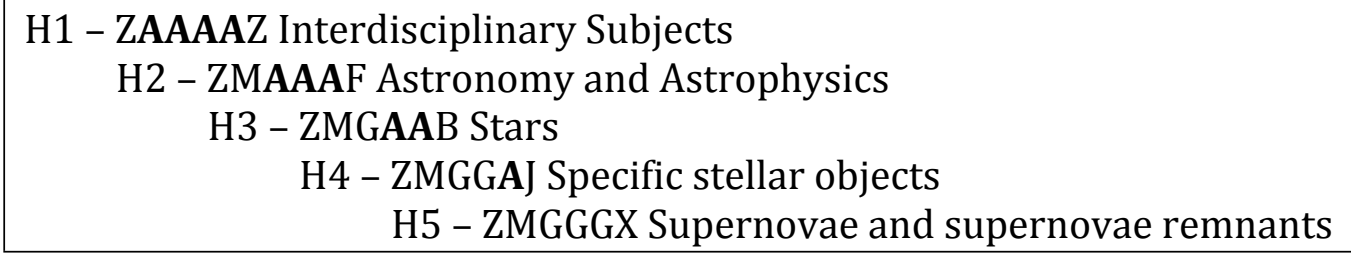

Example 7: Supernovae classification (INSPEC 1973)

The notation applied to the schedule was impractical for subject collocation. The astronomy schedules were displaced from the physics schedules (A-R), and was placed 
at the end of the notational sequence under Z. Eventually, the complexity of applying notation and astronomy's increasing use as a developing discipline area saw it revised into a mainstream schedule within the physics section by 1978 . The newest physical version of the schedule was released in 2004 with the inclusion of geophysics, as seen in Example 8 (INSPEC 2004). The same version of the INSPEC schedule can be found in 1995 (INSPEC 1995) and 1999, and with minor variations in the 1988, 1981-2 and 1978 schedules (Institution of Electrical Engineers 1999; 1988; 1982; 1981; 1978).

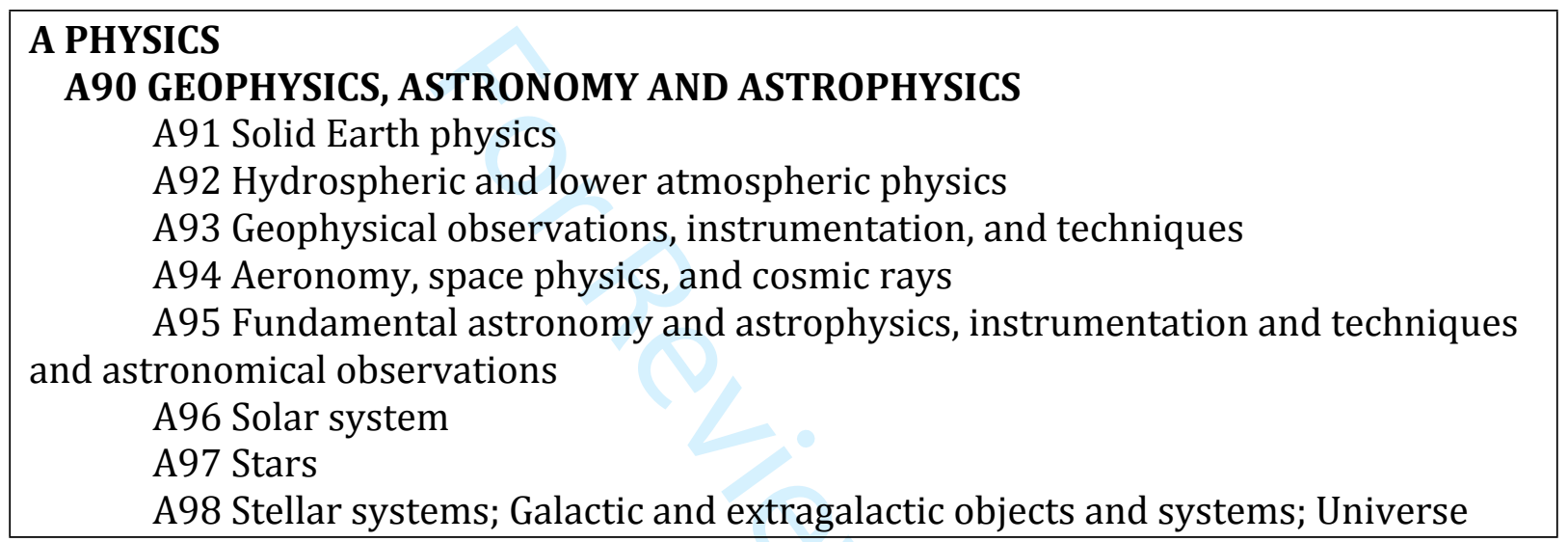
Example 8: Geophysics, astronomy and astrophysics (INSPEC 2004)

The schedule retains five levels of hierarchy; the first level is now the section code letter and the four numbers plus end letter replaces hierarchy levels two to five. It follows that the more specific the number the narrower the search becomes, with the narrowest hierarchy containing a letter suffix to finish the notation (INSPEC 2004, v), as seen in Example 9.

SC (H1) - A Physics

H1 (H2) - A9000 Geophysics, astronomy and astrophysics H2 (H3) - A9700 Stars

H3 (H4) - A9760 Late stages of stellar evolution H4 (H5) - A9760B Supernovae

Example 9: Supernovae classification (INSPEC 2004)

The classification notation changed dramatically over a thirty-year period with fundamental changes to the hierarchical structure of the astronomy and astrophysics schedule. Instead of being placed under 'stars and specific stellar objects' (Example 7), 
supernovae and supernovae remnants were split into subsections for 'stars and late stages of stellar evolution' (Example 9), and 'stellar systems; galactic and extragalactic objects and systems; universe and interstellar medium; nebulae' (Example 10).

\section{SC(H1) - A Physics}

H2 - A9000 Geophysics, astronomy and astrophysics

H3 - A9800 Stellar systems; Galactic and extragalactic objects and systems; Universe

H4 - $\mathbf{A 9 8 4 0}$ Interstellar medium; nebulae H5 - A9840N Supernova remnants

Example 10: Supernova remnants (INSPEC 2004)

Using the example of supernovae and supernovae remnants, it can be seen over time that the INSPEC schedules have tried to cater towards discipline developments. The scheme has been adapted to consider the context in which supernovae are studied either within the context of stellar evolution (Example 9) or as a by-product of stellar evolution and their place within the Universe (Example 10). The order of the subsections was rearranged into a more user-friendly sequence and notation changed to fit in with the other main classes. This contextual flexibility is not seen in the universal schemes.

\subsection{Classification qualities}

The qualities INSPEC displays are typical of an analytico-synthetic scheme; being hierarchical in nature, providing set notation as well as displaying faceted principles, where subject areas are contextually linked and specific, including an index of specific subjects. Outlining the qualities displayed by INSPEC facilitates thoughts on classification principles that could improve its functionality. The qualities are based on Berwick Sayers $(1955,60)$ ideal qualities of a classification scheme (brief, simple and flexible); expressiveness, mnemonic, simplicity, uniqueness, brevity, flexibility and hospitability (see Table 1 below). 


\begin{tabular}{|c|c|}
\hline Expressiveness & $\begin{array}{l}\text { The basic hierarchical structure facilitates narrow and broad } \\
\text { searching. Subsections display fewer relationships between topics on } \\
\text { the same level, but a general order is established based on prominence } \\
\text { within the subject area. Could be improved by reworking subsections } \\
\text { into a specific order relevant to the context of the subject area. }\end{array}$ \\
\hline Mnemonic & $\begin{array}{l}\text { Follows set notational rules. The index provides access to subject } \\
\text { notation making it easy for the classifier to find specific subject } \\
\text { notations. Could improve memorability by providing shorter mixed } \\
\text { notation, including elements of literal mnemonics. }\end{array}$ \\
\hline Simplicity & $\begin{array}{l}\text { Lacks the simplicity of notation needed for ease of retrieval in physical } \\
\text { library environments, but notation has become more simplistic and } \\
\text { easier to understand. Based on a hierarchy, it is appropriate when } \\
\text { determining collocation on shelves or in databases, but could be } \\
\text { adapted to reduce notation length. }\end{array}$ \\
\hline Uniqueness & $\begin{array}{l}\text { Uniqueness for each hierarchal level of the subject. Notation could be } \\
\text { improved by providing shorter notation for general subjects and } \\
\text { longer notation for specific subjects, thus providing clear distinction } \\
\text { between levels of notation and easing confusion when applying } \\
\text { notation. }\end{array}$ \\
\hline Brevity & $\begin{array}{l}\text { Mixed notation of letters and numbers. A maximum of two letters and } \\
\text { four numbers used in a notation, with letters providing the first and } \\
\text { last points in the notational sequence. Provides a huge array of } \\
\text { notational fields and notational flexibility but could be improved by } \\
\text { applying shorter notation for general subject areas. }\end{array}$ \\
\hline Flexibility & $\begin{array}{l}\text { Provides many subject areas in which to classify from and specific } \\
\text { subjects can be chosen without impacting on the rest of the scheme. } \\
\text { Building notation cannot be achieved, leading to less flexibility within } \\
\text { individual library settings for specific or interdisciplinary subjects. }\end{array}$ \\
\hline Hospitality & $\begin{array}{l}\text { Can be easily expanded for the inclusion of new subject areas, object } \\
\text { types and phenomena. Extension of notation could be undertaken } \\
\text { under each main sub-heading as they are yet to fill all the subfields, } \\
\text { and also under the main heading A99. However, expansion as with } \\
\text { enumerative schemes may disrupt the order of the scheme. }\end{array}$ \\
\hline $\begin{array}{l}\text { Notational } \\
\text { structure }\end{array}$ & $\begin{array}{l}\text { Lacks comprehensive faceted auxiliary tables allowing for flexible } \\
\text { notation building. INSPEC is set rather than flexible and subject } \\
\text { coverage is wide-ranging although rather imprecise. Provides for } 2174 \\
\text { notational fields within the Physics section alone, with } 389 \text { of those } \\
\text { applying to the Geophysics, Astronomy and Astrophysics schedule } \\
\text { (INSPEC, 2004). There is still room for expansion, as many notational } \\
\text { fields have yet to be created and used. Notational structure of this } \\
\text { scheme could be improved. }\end{array}$ \\
\hline
\end{tabular}

Table 1: Classification qualities of INSPEC and improvements

\subsection{Findings}

The INSPEC classification has provided evidence that faceted principles can help serve the discipline of astronomy and has facilitated analysis of faceted notational improvements which could increase the scheme's quality. The case study has highlighted 
the importance of hierarchy and providing multiple classifying options for contextual flexibility. The notational element itself is concise but complicated. An adaptation of the notation by removing unnecessary length in general subject areas would allow for ease of application and retrieval. A solution providing for collocation, ease of retrieval and application, and brevity and flexibility could be to include a separate key terms and astronomical object auxiliary table, enabling tagging to take place. The tags would then provide searchable access points to other resources. Although relatively unusual, this would allow for notation to stay simple, flexible and hospitable. Recent research has focused on developing tagging systems to improve search (see, for example, Mendes et al (2009), on improving the library catalogue through the inclusion of tags; Lawson (2009), on the potential of social tagging to enhance traditional subject cataloguing; Pera, Lund and Ng (2009), who developed EnLiS, a library system that improves user searches by using folksonomies to perform similarity matches between keywords in the query and user tags from LibraryThing improving the relevance of results; and Hedden (2008), who advocates the use of semantic tagging that links tags into meaningful taxonomies), so incorporating tagging would not be out of line with current and developing practices. More traditionally, the application of notation building features would provide specific notations allowing for subject complexity.

Broughton $(2006 ; 2015)$ has argued of the importance of faceted classification by supporting its development and heralding it as the future of information retrieval and Kumbhar $(2012,11)$ advises that the best way to organise bibliographic material is through a faceted classification scheme based on scientific principles. Other studies of faceted classification in information retrieval include Mills' (2004) Library Trends paper on faceted classification and logical division in information retrieval, La Barre's (2006) 
PhD thesis, Gnoli and Mei's (2006) study of freely faceted classification for web based retrieval, Uddin and Janacek's (2007) development of a multidimensional classification system in the web that can provide an alternative but convenient structure for organising and finding information content; and Tunkelang's (2009) lecture on using faceted search to provide more effective information seeking support to users in online search systems. It is with this evidence base in mind, and the application of the notational improvements above and use of UAT vocabulary, that development of a new faceted astronomy classification is undertaken.

\section{Development of a faceted astronomy classification}

\subsection{Principles of faceted classification}

The main object of faceted classification is facet analysis. Vickery $(1960,13)$ describes three main steps of facet analysis in the construction of faceted classification schemes: "(i) to assign an order in which the facets will be used in constructing compound subject headings, (ii) to fit the schedules with a notation which permits the fully flexible combination of terms that is needed that is needed and which throws subjects into a preferred filing order, and (iii) to use the faceted scheme in such a way that both specific reference and the required degree of generic survey are possible."

It begins with the creation of facets, the analysis of specific aspects of a subject. Ranganathan developed five facets, each to be applied within the stated order; 1 ) Personality, 2) Matter, 3) Energy, 4) Space and 5) Time, also known as PMEST (Broughton 2015). Personality describes the specific subject within the item to be classified; Matter the properties or material of the subject matter; Energy the processes of the subject; Space the positioning or location of the subject and Time the date of the subject matter. 
Vickery $(1960,30)$ adapted Raganathan's facets into ten new facets; 1) Substance, product, organism, 2) Part, organ, structure, 3) Constituent, 4) Property and measure, 5) Object of action, raw material, 6) Action, operation, process, behaviour, 7) Agent, tool, 8) General property, process, operation, 9) Space and 10) Time. Facets can be specific or broad, simple or complex and can cover almost any aspect of a subject.

Once facets have been chosen for a subject area they need to be split into foci, the second level of subject analysis and isolates, the third level of subject analysis, to get the basic structure of the scheme. Foci are aspects of a facet and isolates are grouped into foci. The foci are then ordered into a suitable arrangement for the subject area, known as order in array (Rowley and Farrow 2000; Rowley and Hartley 2008, 182). Batley $(2005,122)$ lists four possible arrangements of foci; logical, procedural, chronological and alphabetical. Ranganathan $(1937,42-43)$ suggested any order based on systematic principles, such as quantitative, developmental, spatial or time (evolutionary) and canonical. It can be supposed that the arrangement of foci within the facets will be dependent on the main use and subject of the scheme.

Once the basic structure of the scheme is developed, the schedule order and notation is established. Schedule order can either be specific before general, or inverted, so general subject areas come before specific topics, known as the principle of inversion (Rowley and Farrow 2000, 204). Ranganathan proposed the use of inverted order for schedules and notation in faceted classifications. To create notation flexibility, subdivisions from different facets may be joined together to create unique call numbers. This process involves applying a syntax to develop order within the arrangement of facets, helping to aid retrieval. The use of punctuation symbols by Ranaganathan in the CC (, Personality - ; 
Matter - : Energy - . Space - ' Time) provided a syntax which allowed compound subjects to be constructed and facets to be linked together. The citation order of the facets is inverted with personality, matter and energy increasing in specificity and filed before the common facets space and time (Maltby 1975, 62-63). This study constructs a faceted astronomy classification using these classification principles.

\subsection{Classification design}

The first task is to create the relationship facets used to achieve the specifics of notation building. These facets are the 'add-on' themes which can be applied to most subject areas. The facets used in the development of this scheme are influenced by Ranganathan's PMEST and reflect the complex range of information in astronomy classification (see Table 2 below).

\begin{tabular}{|l|l|l|}
\hline Set Facet & Material Type & $\begin{array}{l}\text { The items physical state i.e. Book (BK). Used for every } \\
\text { classification to allow for filing order by material type. }\end{array}$ \\
\hline Facet & Object & $\begin{array}{l}\text { The individual astronomical object. Comprised of } \\
\text { astronomical object catalogues such as Messier and } \\
\text { planet/object type abbreviations i.e. M13 (Globular } \\
\text { Cluster). }\end{array}$ \\
\hline Facet & Process & $\begin{array}{l}\text { The active process present in the work/object. } \\
\text { Comprised of physical processes from physical sciences } \\
\text { i.e. CYV (Cryovolcanism). }\end{array}$ \\
\hline Common Facet & Location & $\begin{array}{l}\text { The apparent location of an astronomical object i.e. M13 } \\
\text { - 16h41m 41.6s +36d27m41s. Comprised of a list of } \\
\text { astronomical right ascension and declination } \\
\text { measurements based on the J2000 equatorial coordinate } \\
\text { system numerically coded to produce shorter notation. }\end{array}$ \\
\hline Common Facet & Time & $\begin{array}{l}\text { The year of observation, discovery or first publication i.e. } \\
\text { Discovery: 1596. Location and Time, will only be used to } \\
\text { distinguish between other works with the same notation } \\
\text { or for specificity. }\end{array}$ \\
\hline
\end{tabular}

Table 2: Relationship Facets

Each relationship facet will have its own auxiliary table providing coded notation for the terms, objects and dates. The set facet is the beginning notation allowing for the division of material types. The remaining facets are added after the subject schedule notation. 


\subsubsection{Subject schedules for astronomy}

Astronomy is a broad and complex interdisciplinary subject. To understand its complexities, a list of the most common subject areas within taught astronomy was drawn from current textbooks (see Vickery (1960 20) for more on this methodological step). Five textbooks demonstrating universal astronomy topics were chosen and their table of contents analysed for key and repetitive subject areas. The chapter introductions and summaries were discarded to keep the topic investigation clear. Table 3 shows the main topic areas found.

\begin{tabular}{|l|l|}
\hline Solar System & Sun and Stars \\
Terrestrial Planets & Sun: surface, interior and atmosphere \\
Giant Planets (Gas and Ice) & Stars: measurement and observations \\
Asteroids, Kuiper Belt and Comets & Stellar Formation \\
Planetary formation & Main Sequence Life \\
Geological and surface processes & Stellar Death \\
Atmospheric processes & Stellar Remnants \\
& (Green and Jones 2015) \\
(McBride and Gilmour 2004) & Planetary Science \\
Galaxies and Cosmology & Solar System: composition, formation and \\
Milky Way composition & dynamics \\
Galaxy: classification, formation and evolution & Solar: energy transfer, atmosphere and surface \\
Normal Galaxies & Planetary: atmospheres, surfaces and interiors \\
Active Galaxies & Magnetic Fields \\
Galaxy distribution & Other Solar System Bodies: meteorites, minor \\
Universe: evolution, measurement and problems & planets, comets and planetary rings \\
& Extrasolar Planets \\
& Planetary Formation \\
& \\
(Jones, Lambourne and Serjeant 2015) & (de Pater and Lissauer 2015) \\
\hline $\begin{array}{l}\text { Universe } \\
\text { Astronomy: observation and equipment } \\
\text { Planets and Moons: terrestrial, gas and ice } \\
\text { Stars: formation, evolution, death and oddities } \\
\text { Galaxies: Milky way, normal and active } \\
\text { Cosmology: origins, evolutional and SETI }\end{array}$ \\
\begin{tabular}{|l} 
(Freedman and Kaufmann 2016) \\
Table 1: Main Topic Areas within Astronom
\end{tabular} \\
\hline
\end{tabular}

Table 1: Main Topic Areas within Astronomy Textbooks 
The topic investigation also found a new classification system created by Dick (2013) demonstrating a kingdom, family and class classification structure for the classification of astronomy objects, based on hierarchical science kingdom classification systems. The three main kingdoms are: Planets, Stars and Galaxies. The kingdoms are arranged hierarchically, increasing in size and complexity. Comparing the hierarchical kingdom classification with the topic investigation results found an overlap in subject matter. Grouping the main topic areas within astronomical textbooks with Dick's classification structure of astronomy objects produced four main subject areas, and examining current schedules for DDC, UDC and INSPEC produced two minor subject areas (see Table 4 below).

\section{Main Divisions \\ Planetary Systems \\ Stellar Systems \\ Galactic Systems \\ Cosmology}

\section{Minor Divisions}

Astronomical Techniques

Astronomical Equipment

Table 4: Main and Minor divisions

The main subject divisions increase in evolutionary scale from localized phenomena and objects to the origins and evolution of the Universe. The minor subject divisions cover the techniques and equipment used in astronomical observation. These six subject areas form the knowledge divisions of astronomy and each will form their own subject schedule. In addition to building classification from the subject schedules and relationship facets, the scheme should ideally cater for subject searching via controlled subject headings.

\subsubsection{Unified Vocabulary}


The UAT, a free resource delivering internationally unified astronomical terminology, is constantly revised by scientists and astronomers providing unified subject headings. The use of UAT subject headings in this classification scheme allows for scientific accuracy within subject areas and narrower and broader searching. As of 2015, the UAT had 1,906 terms, a range of 12 hierarchical levels and 15 top level concepts (Frey et al. 2015). On the release of UAT v.1, the scheme was updated to include 1,834 terms, a range of 10 hierarchical levels and 11 top level concepts (Unified Astronomy Thesaurus 2017a). The current structure of the thesaurus has changed in the intervening period with the terminology being equally distributed over the top-level concepts. The release of UAT v.2.0.0 in early 2017 cleaned up subject duplications and provided 16 new terms (Unified Astronomy Thesaurus 2017b). This resource is necessary for this scheme's interoperability.

\subsubsection{Notation Building}

Literal mnemonics will be used to satisfy Berwick Sayer's ideal notational qualities and each main and minor division will start with a defining letter code which is representative of the subject area (see Table 5 below).

\section{Main Divisions}

$$
\begin{array}{ll}
\text { P } & \text { Planetary Systems } \\
\text { S } & \text { Stellar Systems } \\
\text { G } & \text { Galactic Systems } \\
\text { C } & \text { Cosmology }
\end{array}
$$

\section{Minor Divisions}

AT Astronomical Techniques

AE Astronomical Equipment

Table 5: Coded Main and Minor divisions

The main and minor divisions are subdivided three times to produce a sequence of three numbers, each with its own hierarchy. Three subdivisions enable a certain level of subject 
specificity which can be further quantified using the relationship facets. This produces a simple and memorable three-digit code at its maximum which is then applied to the subject letter code i.e. P111. The ending notation is based on the specificity of the work and the application of the relationship facets. The relationship facet notation varies between letter suffixes and numerical codes providing unique notation in most cases. Material type is denoted by a two-letter abbreviation; object by its catalogue code or three-letter abbreviation; process by a three-letter abbreviation; location by a two-digit code and time by its four-number year notation (see 5.3 Provisional astronomy classification for details). The application of these facets is dependent on the specificity of the work and material type; however, simplicity and uniqueness can still prevail by using only the material type abbreviation, division code and three-digit subject code.

To synthesise the facets together, a syntax of notational punctuation will be used. The notational punctuation used to display the faceted relationships are built from mathematical and literary punctuation and have been influenced by Ranganathan's work (see Table 6 below).

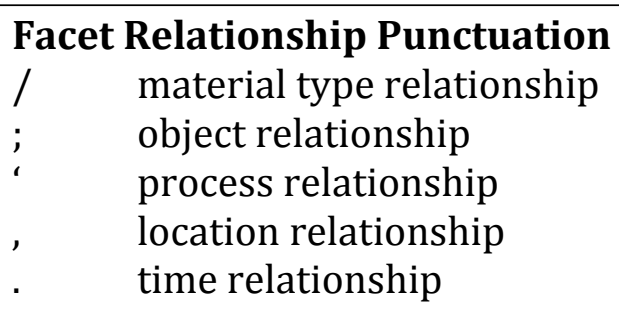

\section{Subject Relationship Punctuation}

$=\quad$ equal knowledge of two or more subject areas

$+\quad$ more than equal knowledge of two or more subject areas

- $\quad$ less than equal knowledge of two or more subject areas

: $\quad$ astronomical technique or equipment

Table 6: Punctuation for facets and subject relationships

The schedule order is based on Ranganathan's inverted principle for faceted classification, whereby general facets are filed before specific facets; the order being 
material type, object, process, location and time. The facet for material type is crucial for the effective filing and retrieval of an item within a physical or online library environment and is added to the beginning of the call number. The other relationship facets are added after the subject notation based on increasing specificity. The use of mixed notation in the creation of a call number allows for Berwick Sayers ideals of classification to be upheld and for this scheme to have a modern classification approach. As will be seen in the examples below, the scheme's main disadvantage is that compound subjects can create complex notation making shelf arrangement challenging; though the application of complex notation is decided by the classifier and can therefore be negated.

\subsubsection{Examples of classification}

Reviewed below is an example outline of a main knowledge division in the subject schedules and an interpretation of classifying a book, paper and observation using the basic schedules and relationship facets. They display the usability and functionality of the classification scheme for different material types and subject areas. The schedule order is hierarchical, based on Dick's (2013) kingdom, family and class classification and then further ordered spatially if applicable i.e. Solar System planets.

\begin{tabular}{|lc|}
\hline Main Division & P Planetary Systems \\
Subdivision of Main Division & P1 Solar System \\
Second Subdivision of Main Division & P11 Planetary Types \\
Third Subdivision of Main Division & P111 Terrestrial Planets
\end{tabular}

Example 11: Main knowledge division

Planet Mercury: from pale pink dot to dynamic world, by David A. Rothery.

BK

P Planetary Systems

P1 Solar System

P11 Planetary Types

P111 Terrestrial Planets

Relationship Facets 
Object: Mercury $\Rightarrow$;MER

Process: Geology $\Rightarrow$ 'GEO

Notation: BK/P111;MER'GEO

Example 12: Class mark for a book

Cepheid variables in the flared outer disk of our galaxy, by Michael W. Feast, John W. Menzies, Noriyuki Matsunaga and Patricia A. Whitelock. Nature, 2014, Vol.509(7500), p.342.

JA

S Stellar Systems

S3 Variable Stars

S31 Intrinsic Variables

S311 Pulsating Variables

G Galactic Systems

G5 Milky Way

Relationship Facets

Object: Cepheid Variables $\Rightarrow$;CEV

Notation: JA/S311=G5;CEV

Example 13: Class mark for an article

2015 Radio observation of spiral galaxy M61

DP

G Galactic Systems

G1 Galaxies

G11 Galaxy Types

G113 Spiral Galaxies

AT Astronomical Techniques

AT1 Observation Methods

AT13 Radio

Relationship Facets

Object: M61 $\Rightarrow$;M61

Time: $2015 \Rightarrow .2015$

Notation: DP/G113:AT13;M61.2015

Example 14: Class mark for a physical dataset: observation

5.3 Provisional astronomy classification 


\section{Provisional Astronomy Classification Subject Schedules}

Subject Schedules - Planetary Systems (P), Stellar Systems (S), Galactic Systems (G), Cosmology (C), Astronomical Techniques (AT), Astronomical Equipment (AE).

Facets - Material Type, Object, Process, Location, Time.

Material Type - Book (BK), Journal Article (JA), Sky Atlas' (SA), Dataset - Physical (DP), Dataset - Digital (DD), Photographic Plates (PP), Equipment (EQ).

Object - Messier catalogue, Planets, Moons, Stellar Types, Galactic Types.

Process - Planets: Formation Processes, Surface and Interior Geology, Atmospheric Processes. Stars: Formation Processes, Core Reactions, Atmospheric Processes.

Galaxies: Formation Processes.

Location - RA \& Dec Measurements: Northern Hemisphere ( $\mathrm{Dec}$, 仓RA) and Southern Hemisphere ( $\preccurlyeq$ Dec, $\preccurlyeq R A)$.

Time - Date Ranges: BC, 0-500, 501-1000, 1001-1500, 1501-2000, 2001-2500.

\section{Facet Relationship Punctuation}

/ material type relationship

; $\quad$ object relationship

- process relationship

, location relationship

. time relationship

\section{Subject Relationship Punctuation}

$=\quad$ equal knowledge of two or more subject areas

$+\quad$ more than equal knowledge of two or more subject areas

- $\quad$ less than equal knowledge of two or more subject areas

: $\quad$ astronomical technique or equipment

\section{Basic Draft Schedules}

\section{P Planetary Systems}

P1 Solar System

P11 Planetary Types

P111 Terrestrial Planets

P112 Gas Giant Planets

P113 Ice Giant Planets

P12 Planetary Features

P121 Moons/Satellites

P122 Rings

P123 Radiation Belts

P13 Solar System Objects

P131 Dwarf Planets

P132 Meteoroids 
P133 Asteroids

P134 Comets

P135 Trans-Neptunian Objects

P14 Solar System Regions

P141 Asteroid Belt

P142 Kuiper Belt

P143 Oort Cloud

P2 Extrasolar Planets

P3 Planetary Formation

P31 Protoplanetary Disk

P32 Planetary Collisions

P33 Planetary Migration

P4 Interplanetary Medium Features

P41 Gas

P42 Dust

P43 Solar Wind

P44 Cosmic Rays

P5 Astrobiology

\section{S Stellar Systems}

S1 Stellar Sequence

S11 Pre-Main Sequence Stars

S112 Protostars

S12 Main Sequence Stars

S121 0 Class

S122 B Class

S123 A Class

S124 F Class

S125 G Class

S124 K Class

S125 M Class

S13 Post-Main Sequence Stars

S131 Subgiant Class

S132 Giant Class

S133 Bright Giant Class

S134 Supergiant Class

S135 Hypergiant Class

S14 Stellar Evolution-Death

S141 Supernovas

S142 Novae

S143 White Dwarfs

S144 Neutron Stars

S145 Black Holes

S146 Planetary Nebula

S15 Stellar Remnants

S151 Supernova Remnants

S152 Nova Remnants

S153 Planetary Nebula Remnants

S2 Multiple Star Systems 
S21 Binary Stars

S211 Brown Dwarfs

S22 Multiple Stars

S23 OB Associations

S24 Stellar Clusters

S241 Open Clusters

S242 Globular Clusters

S3 Variable Stars

S31 Intrinsic Variables

S311 Pulsating Variables

S312 Eruptive Variables

S313 Cataclysmic Variables

S32 Extrinsic Variables

S321 Rotating Variables

S322 Eclipsing Binaries

S323 Planetary Transits

S4 Exotic Stars

S41 Quark Stars

S42 Boson Stars

S43 Electroweak Stars

S44 Preon Stars

S45 Planck Stars

S5 Interstellar Medium Features

S51 Dust

S52 Gas

S521 H I Cloud

S522 H II Cloud

S523 H 2 Cloud

S53 Stellar Wind

S54 Galactic Cosmic Rays

\section{G Galactic Systems}

G1 Galaxies

G11 Galaxy Types

G111 Elliptical Galaxies

G112 Lenticular Galaxies

G113 Spiral Galaxies

G114 Irregular Galaxies

G115 Barred Galaxies

G116 Dwarf Galaxies

G12 Active Galaxies

G121 Seyfert Galaxies

G122 Radio Galaxies

G123 Quasars

G124 Blazars

G13 Galactic Features

G131 Galactic Ring

G132 Galactic Accretion Disk 
G133 Galactic Jets

G134 Galactic Halos

G2 Multiple Galaxy Systems

G21 Binary Galaxies

G22 Interacting Galaxies

G23 Galactic Clusters

G24 Galactic Superclusters

G3 Galaxy Formation

G31 Galaxy Mergers

G4 Intergalactic Medium Features

G41 Gas

G42 Dust

G43 Galactic Wind

G44 Extragalactic Cosmic Rays

G5 Milky Way

\section{Cosmology}

C1 The Universe

C11 Origins

C12 Evolution

C13 Age

C131 Hubble Time

C14 Temperature

C2 Cosmic Matter

C141 Black-body Spectrum

C21 Baryonic Matter

C211 Hydrogen Plasma

C212 Helium Plasma

C22 Dark Matter

C221 Baryonic Dark Matter

C222 Non-baryonic Dark Matter

C23 Cosmic Density/Energy

C231 Dark Energy

C232 Dark Energy Density

C3 Cosmic Radiation

C31 Electromagnetic Radiation

C311 Radio waves

C312 Microwaves

C313 Infrared Radiation

C314 Visible Light

C315 Ultraviolet Radiation

C316 X-Rays

C317 Gamma Rays

C32 Cosmic Microwave Background

C33 Cosmic Background Radiation

C4 Cosmic Expansion

C41 Hubble's Law

C411 Hubble Flow

C412 Hubble Constant 
C413 Hubble Parameter

C42 Redshift

C43 Blueshift

C5 Cosmological Models

C51 Special Relativity

C511 Space-Time

C512 Time Dilation

C52 General Relativity

C521 Curvature of Space-Time

C522 Einstein's Field Equations

C53 Cosmological Principle (Uniformity)

C54 Einstein Model

G55 de Sitter Model

G56 FRW Models

G57 Einstein-de Sitter Model

G58 Eddington-Lemaître Model

G59 Lemaître Model

C6 Cosmological Theories

C21 Big Bang

C22 Big Crunch

C23 Multiverses

C24 Rebound Theory

C25 String Theory

C7 SETI

AT Astronomical Techniques

AT1 Observation Methods

AT11 Photometry

AT12 Spectroscopy

AT13 Radio

AT14 Infrared

AT15 Gamma Ray

AT16 X-Ray

AT17 Microwave

AT18 Ultraviolet

AT2 Distance Measurements

AT21 Trigonometric Parallax

AT22 Spectroscopic Parallax

AT23 Doppler Shift

AT24 Hubble's Law

AT3 Luminosity Measurements

AT31 Apparent Magnitude

AT32 Absolute Magnitude

AE Astronomical Equipment

AE1 Observatories 


\begin{tabular}{|c|}
\hline AE11 Earth Observatories \\
AE12 Space Observatories \\
AE2 Telescopes \\
AE21 Optical Telescopes \\
AE211 Refracting Telescope \\
AE212 Reflecting Telescope \\
AE213 Catadioptric Telescope \\
AE22 Non-Optical Telescopes \\
AE221 Radio Telescope \\
AE222 X-Ray Telescope \\
AE223 Gamma Ray Telescope \\
AE224 Infrared Telescope \\
AE225 Ultraviolet Telescope \\
\end{tabular}

\subsection{Proposed use of classification scheme}

The scheme's author has envisaged the classification scheme in an accessible online format, whereby institutions hold an online subscription much like WebDewey and LCC's Classification Web. Each subject schedule could be searched hierarchically through an expandable linked list, thereby making it easy to go from the broadest term to the narrowest whilst retaining the steps taken. Ideally, the scheme would allow for a split screen mode, meaning classifiers could search as many subject schedules as needed to find the subject codes for the work. This would be advantageous not only for comparison of subjects for non-experts but also for applying subject codes to interdisciplinary works. The relationship facets (Material Type, Object, Process, Location, Time) would have their own tabs under Set Facet, Facet and Common Facet, with an expansion list of coded notation in numerical or alphabetical order. Furthermore, a keyword search function on each page would provide intuitive manipulation of the lists for ease of searching. A feature which could be integrated into the online scheme is of a classification checker, whereby the classifier could input the main classificatory features of an item (drop down lists provided) into a 'classification calculator' (see mock up below). The scheme would then automatically arrange the information based on the classifications rules and add in 
relevant relationship punctuation to form a list of feasible class marks for the item. The classifier would then be able to double check the class marks and ascertain their suitability within their collection. Other tabs in the online format would hold key information on how to build and check a class mark as well as information on improvements and revised subject schedules. Feedback through an online form, as well as an 'Ask a librarian' feature would enable classifiers to directly feedback to the systems creators allowing for developments to occur quicker than in other traditional classification schemes.

Subject 1: Material Type: Process:

Subject 2: Object 1: Location:

Subject 3: Object 2: Time:

[Search Classification]

Class mark 1:

Class mark 2:

Example 15: Mock up of 'classification calculator' for astronomy classification

\section{Concluding comments}

The discipline of astronomy requires its own classification scheme for several reasons. Firstly, the provision of astronomy schedules within current universal schemes lacks the specificity for complex subject classification. Secondly, special classification schemes built for astronomy have either been merged with physics-based schemes or are too simple to provide useful notation in environments with multiple material types. And thirdly, the lack of flexibility within these schemes means there isn't a comprehensive interdisciplinary scheme for use in astronomical libraries. Developing a special 
classification scheme on faceted classification principles provides a specific and flexible classification catering to the discipline's interdisciplinary nature. Adding unified terminology in the form of broader and narrower terms through UAT enhances the usability of a new scheme within astronomy libraries.

This study has provided the means of investigating an overlooked discipline area within librarianship discourse and has provided an insight into astronomy classification and its relationship within classification types. Understanding the nature of the discipline of astronomy and how it can be provided for within library classification schemes is key to the continuation and future development of astronomy classification for specialist collections. Further research that could be undertaken consists of revising, finishing and testing the classification development within a physical library setting, where its flexibility and functionality can be analysed and the scheme improved.

TOTAL WORDS: 8445

References

Access Innovations Inc. 2018. “Access Innovations, Inc. and American Institute of Physics “Unravel” New Thesaurus for Online Scholarly Publications,"

https://www.accessinn.com/access-innovations-inc-and-american-institute-of-physicsunravel-new-thesaurus-for-online-scholarly-publications/.

Accomazzi, Alberto, Norman Gray, Chris Erdmann, Chris Biemesderfer, Katie Frey, and Justin Soles. 2014. “The Unified Astronomy Thesaurus.” Paper presented at the Conference Astronomical Data Analysis Software and Systems XXIII, Waikoloa Beach 
Marriott, Hawaii, USA. 29 September - 3 October, 2014. ASP Conference Series 485: 461464.

BARTOC.org. 2017. “International Virtual Observatory Alliance Thesaurus,”

\section{https://bartoc.org/en/node/381.}

Batley, Susan. 2005. Classification in Theory and Practice. Oxford: Chandos.

Berwick Sayers, William Chadwick. 1955 A Manual of Classification for Lbrarians and Bibliographers. 3rd revised ed. London: Grafton \& Co.

British Sociological Association. 2002. "Statement of Ethical Practice for the British Sociological Association," https://www.britsoc.co.uk/equality-diversity/statement-ofethical-practice/.

British Standards Institution. 2005. UDC, Universal Decimal Classification: Volume 1 Systematic Tables. 3rd ed. London: British Standards Institution.

Broughton, Vanda. 2006. “The Need for a Faceted Classification as the Basis of all Methods of Information Retrieval." Aslib Proceedings 58, nos. 1/2: 49-72. doi: $10.1108 / 00012530610648671$

Broughton, Vanda. 2015. Essential Classification. 2nd ed. London: Facet Publishing. Chowdhury, Gobinda. 2010. Introduction to Modern Information Retrieval. 3rd ed. London: Facet Publishing.

Chowdhury, Gobinda G., and Sudatta Chowdhury. 2007. Organizing Information: From the Shelf to the Web. London: Facet Publishing.

CILIP. 2015. "Code of Professional Practice,"

https://www.cilip.org.uk/about/ethics/code-professional-practice.

Corbin, Brenda. 2003. "The Evolution and Role of the Astronomical Library and Librarian." In Information Handling in Astronomy: Historical Vistas. Astrophysics and 
Space Science Library 285, edited by A. Heck, 139-155. New York: Kluwer Academic Publishers.

Crovisier, Ronald, and Sheila S. Intner. 1987. "Classification for Astronomy: the QB Schedule of the Library of Congress Classification." Cataloguing \& Classification Quarterly 7, no. 3: 23-36. doi: 10.1300/J104v07n03_04

De Pater, Imke, and Jack J. Lissauer. 2015. Planetary Sciences. Updated 2nd ed. Cambridge: Cambridge University Press.

Dick, Steven J. 2013. Discovery and Classification in Astronomy: Controversy and Consensus. Cambridge: Cambridge University Press.

Field, Barry James. 1973. INSPEC Classification: A Classification Scheme for Physics, Electrotechnology, Computers and Control. Institution of Electrical Engineers. Freedman, Roger. and William J. Kaufmann. 2016. Universe. 10th ed. New York: W.H. Freeman and Co.

Frey, Katie. Christopher Erdmann and Alberto Accomazzi. 2015. "Management of the Unified Astronomy Thesaurus." Paper presented at the Conference Library and Information Services in Astronomy VII: Open Science at the Frontiers of Librarianship, Naples, Italy. June 17-20, 2014. ASP Conference Series, 492: 204-207.

Gnoli, Claudio, and Hong Mei. 2006. "Freely Faceted Classification for Web-based Information Retrieval." New Review of Hypermedia and Multimedia 12, no. 1: 63-81. Green, Simon and Mark Jones, 2015. An Introduction to the Sun and Stars. 2nd ed. Cambridge: Cambridge University Press.

Heck, Andre, 2003. Information Handling in Astronomy: Historical Vistas. Astrophysics and Space Science Library, 285. New York: Kluwer Academic Publishers.

Hedden, Heather. 2008. "How Semantic Tagging Increases Findability.” EContent 31, no. 8: 38-43. 
Herner, Saul. and Robert S. Meyer. 1957. “Classifying and Indexing for the Special Library." Science New Series 125, no. 3252: 799-803.

\section{http://www.jstor.org/stable/1754075.}

Hider, Philip. and Ross. Harvey. 2008. Organising Knowledge in a Global Society:

Principles and Practices in Libraries and Information Centres. Topics in Australasian Library and Information Studies, 29. Revised ed. Wagga Wagga: Centre for Information Studies, Charles Sturt University.

Hoskin, Michael. 2003. The History of Astronomy: A Very Short Introduction. Oxford: Oxford University Press

Hunter, Eric J. 2018. Classification Made Simple. 2nd ed. Abingdon: Routledge. Ibekwe-SanJuan, Fidelia. 2008. The Impact of Geographic Location on the Development of a Specialty Field: A Case Study of Sloan Digital Sky Survey in Astronomy. Knowledge Organization, 35, no. 4: 239-250.

INSPEC. 1995. Classification 1995: A Classification Scheme for the INSPEC Database. Institution of Electrical Engineers.

INSPEC. 2004. INSPEC classification 2004. INSPEC.

INSPEC. 1976. Concordance to the INSPEC classification, 1969-1976. London: INSPEC. Institute of Astronomy. 2017. "Dewhirst Classification," http://www.ast.cam.ac.uk/library/guide/dewhirst. Institution of Electrical Engineers. 1999. Classification 1999: A Classification Scheme for the INSPEC Database. INSPEC.

Institution of Electrical Engineers. 1988. INSPEC Classification: A Classification Scheme for Physics, Electrotechnology, Computers, and Control. Institution of Electrical Engineers. 
Institution of Electrical Engineers. 1982. INSPEC Classification: A Classification Scheme for Physics, Electrotechnology, Computers, and Control. Institution of Electrical Engineers.

Institution of Electrical Engineers. 1981. INSPEC Classification: A Classification Scheme for Physics, Electrotechnology, Computers, and Control. Institution of Electrical Engineers.

Institution of Electrical Engineers. 1978. INSPEC Classification: A Classification Scheme for Physics, Electrotechnology, Computers, and Control. Institution of Electrical Engineers.

Jones, Mark, Robert Lambourne. and Stephen Serjeant, eds. 2015. An Introduction to Galaxies and Cosmology. 2nd ed. Cambridge: Cambridge University Press.

Kumbhar, Rajendra. 2012. Library Classification Trends in the $21^{\text {st }}$ Century. Oxford: Chandos Publishing.

La Barre, Kathryn. 2006. The Use of Faceted Analytico-Synthetic Theory as Revealed in the Practice of Website Construction and Design. PhD diss., Indiana University. Lawson, Karen G. 2009. "Mining Social Tagging Data for Enhanced Subject Access for Readers and Researchers." The Journal of Academic Librarianship 35, no. 6: 574-582. Mills, Jack. 2004. "Faceted Classification and Logical Division in Information Retrieval.” Library Trends 52, no. 3, Winter: 541-570.

Pera, Maria Soledad, William Lund, and Yiu-Kai Ng. "A Sophisticated Library Search Strategy using Folksonomies and Similarity Matching." Journal of the American Society for Information Science and Technology 60, no. 7: 1392-1406.

Ricketts, Sandra, Christina Birdie, and Eva Isaksson. 2007. “Ontologies for Astronomy.” Paper presented at the Conference Library and Information Services in Astronomy V: 
Common Challenges, Uncommon Solutions, Cambridge, Massachsetts, USA. June 18-21, 2006. ASP Conference Series 377:193-196.

Losee, Robert M. 1995. “How to Study Classification Systems and their Appropriateness for Individual Institutions." Cataloguing \& Classification Quarterly 19, nos. 3/4: 45-58. doi: 10.1300/J104v19n03_05

Maltby, Arthur. 1975. Sayers' Manual of Classification for Librarians. 5th ed. London: André Deutsch.

McBride, Neil and Iain Gilmour, 2004. An Introduction to the Solar System. Cambridge: Cambridge University Press.

Mendes, Luiz H., Jennie Quiñonez-Skinner, and Danielle Skaggs. 2009. “Subjecting the Catalog to Tagging." Library Hi Tech 27, no. 1: 30-41.

Ranganathan, Shiyali Ramamrita. 1937. Prolegomena to Library Classification. Madras: Madras Library Association.

Rowley, Jennifer and John Farrow. 2000. Organizing Knowledge: An Introduction to Managing Access to Information. 3rd ed. Aldershot: Ashgate Publishing. Rowley, Jennifer and Richard Hartley. 2008. Organizing Knowledge: an Itroduction to Managing Access to Information. 4th ed. Aldershot: Ashgate Publishing. The Library of Congress. 2017. “Library of Congress Classification PDF files: Q text,” https://www.loc.gov/aba/publications/FreeLCC/Q-text.pdf.

Tunkelang, Daniel. 2009. "Faceted Search." Synthesis Lectures on Information Concepts, Retrieval, and Services 1, no. 1: 1-80.

Nasir Uddin, Mohammad, and Paul Janecek. 2007 "The Implementation of Faceted Classification in Web Site Searching and Browsing." Online Information Review 31, no. 2: 218-233. 
Unified Astronomy Thesaurus. No date. "Hierarchical Browse: Supernovae," Accessed October 13, 2017. http://astrothesaurus.org/thesaurus/hierarchical-browse/. Unified Astronomy Thesaurus. 2017a. "Unified Astronomy Thesaurus v.1 is Here!.” Unified Astronomy Thesaurus (blog). December 23, 2015.

http://astrothesaurus.org/blog/.

Unified Astronomy Thesaurus. 2017b. “Version 2.0.0 of the Unified Astronomy

Thesaurus." Unified Astronomy Thesaurus (blog). January 31, 2017.

http://astrothesaurus.org/blog/.

Vickery, Brian. 1960. Faceted Classification: A Guide to Construction and Use of Special Schemes. London: Aslib.

WebDewey. 2017. "WebDewey,"

http://www.dewey.org/webdewey/standardSearch.html.

Wilkins, George. 1989. “The Revision of the Universal Decimal Classification for Astronomy." International Astronomical Union Colloquium 110: 70-71.

Wilkins, George. 1995. "Revision of UDC 52 for Astronomy: Report on the "Birds of Feather" session at LISA-II." Vistas in Astronomy 39, no. 2: 263-264. 


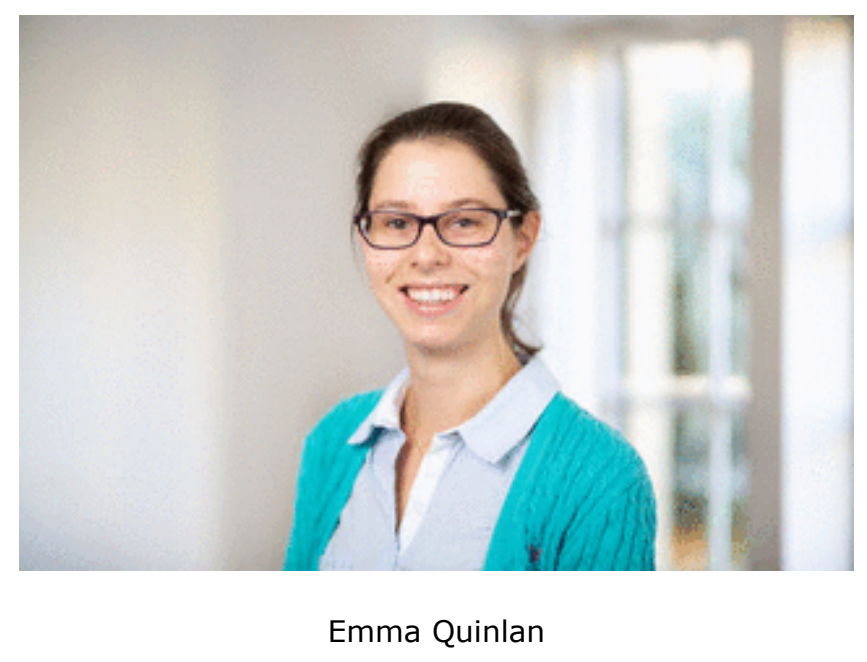

$112 \times 75 \mathrm{~mm}(72 \times 72 \mathrm{DPI})$ 


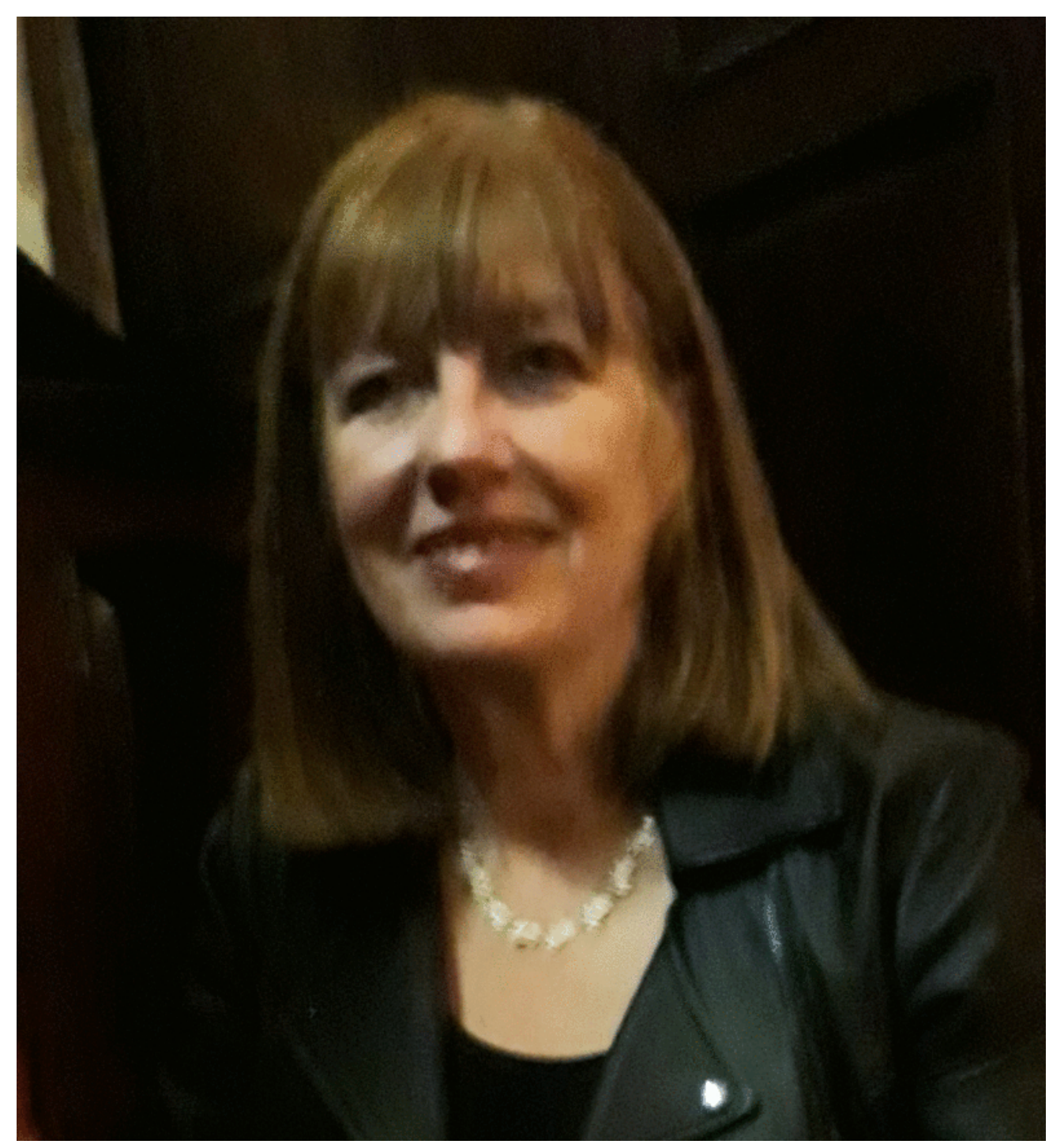

Pauline Rafferty

$280 \times 306 \mathrm{~mm}(72 \times 72 \mathrm{DPI})$ 